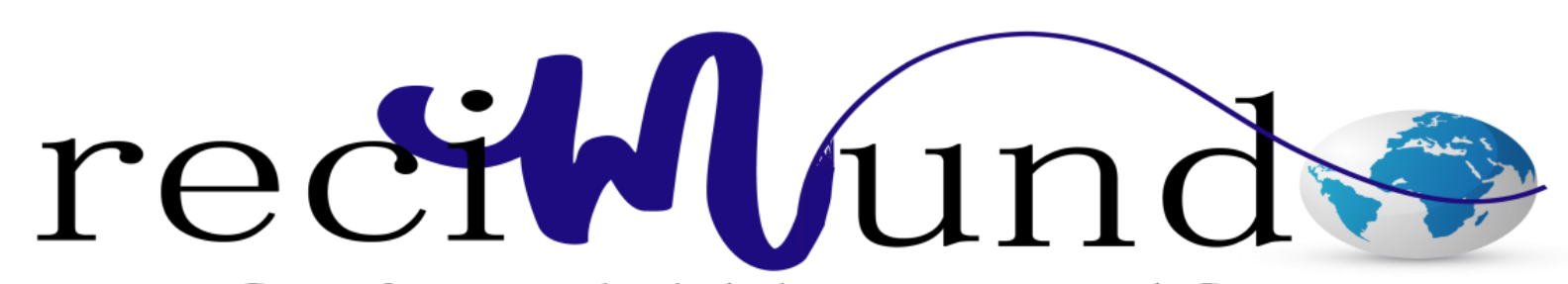

Revista Científica Mundo de la Investigación y el Conocimiento

Joao Andrés Rodríguez Molina a ; Pedro Javier Chong Cevallos ${ }^{\text {b }}$; Julio César Tixe

Peralta ${ }^{c}$; Rogelio Andrés Leyton Acuña ${ }^{\mathrm{d}}$

Tratamiento conservador del esguince de tobillo

Conservative treatment of ankle sprain

Revista Científica Mundo de la Investigación y el Conocimiento. Vol. 3 núm.3. Esp., noviembre, ISSN: 2588-073X, 2019, pp. 421-437

DOI: $10.26820 /$ recimundo/3.(3.Esp).noviembre.2019.421-437

URL: http://recimundo.com/index.php/es/article/view/608

Código UNESCO: 3205 Medicina Interna

Tipo de Investigación: Artículo de Revisión

(C) RECIMUNDO; Editorial Saberes del Conocimiento, 2019

Recibido: 15/09/2019

Aceptado: 23/10/2019

Publicado: 30/11/2019

Correspondencia: joao.andrés.rod@gmail.com

\footnotetext{
a. Médico; Investigador Independiente; Guayaquil, Ecuador; joao.andrés.rod@ gmail.com

b. Médico; Investigador Independiente; Guayaquil, Ecuador; pedrochongc@ gmail.com

c. Médico; Investigador Independiente; Guayaquil, Ecuador; julianotixe@ hotmail.com

d. Médico; Fundación Academia Internacional de Ciencias Empresariales; Investigador Independiente; Guayaquil, Ecuador; $\underline{\text { rogleyton@gmail.com }}$
} 


\section{Tratamiento conservador del esguince de tobillo}

Vol. 3, núm. 3 Esp., (2019)

Joao Andrés Rodríguez Molina; Pedro Javier Chong Cevallos; Julio César Tixe Peralta; Rogelio Andrés Leyton Acuña

\section{RESUMEN}

El esguince de tobillo es una de las lesiones ortopédicas más comunes para la vida diaria de personas sean deportistas o no. Normalmente el diagnóstico es subestimado por el mismo lesionado sin considerar que las lesiones en los ligamentos producen inestabilidad en la postura siempre que no se haga el correcto tratamiento de la lesión. Cuando se hace evidente una afección física, el paciente siempre espera el "mejor escenario" para el tratamiento y consecutiva recuperación. Esta esperanza es confundida con la posibilidad de acceder al "tratamiento conservador”, pero ¿de qué se trata el mismo? En el presente trabajo se pretende encarar esta idea "no invasiva" y sus reales implicaciones en el tratamiento de esguince de tobillo a través de una revisión bibliográfica selectiva de la literatura académica disponible al respecto. Se ha encontrado que el tratamiento siempre será conservador cuando el diagnóstico es el adecuado. Independientemente de que las lesiones más graves requieran de una intervención quirúrgica está visto que alternativas como las fisioterapias, la inmovilización temprana siempre serán parte del plan médico para la recuperación del paciente preventivamente para evitar la cirugía o precisamente para recuperarse de ella.

Palabras Claves: Esguince; Tobillo; Tratamiento; Conservador; Cirugía. 


\title{
Tratamiento conservador del esguince de tobillo
}

Vol. 3, núm. 3 Esp., (2019)

Joao Andrés Rodríguez Molina; Pedro Javier Chong Cevallos; Julio César Tixe Peralta; Rogelio

Andrés Leyton Acuña

\begin{abstract}
Sprained ankle is one of the most common orthopedic injuries for the daily life of people, whether they are athletes or not. Normally the diagnosis is underestimated by the injured person without considering that ligament injuries produce instability in the posture provided that the correct treatment of the lesion is not made. When a physical condition becomes evident, the patient always expects the "best scenario" for treatment and consecutive recovery. This hope is confused with the possibility of accessing "conservative treatment", but what is it about? In the present work, we intend to address this "non-invasive" idea and its real implications in the treatment of ankle sprain through a selective bibliographic review of the available academic literature. The treatment will always be conservative when the diagnosis is appropriate. Regardless of whether the most serious injuries require surgical intervention, it is seen that alternatives such as physiotherapy, early immobilization will always be part of the medical plan for the recovery of the patient preventively to avoid surgery or precisely to recover from it.
\end{abstract}

Keywords: Sprain; Ankle; Treatment; Conservative; surgery. 


\section{Tratamiento conservador del esguince de tobillo}

Vol. 3, núm. 3 Esp., (2019)

Joao Andrés Rodríguez Molina; Pedro Javier Chong Cevallos; Julio César Tixe Peralta; Rogelio Andrés Leyton Acuña

\section{Introducción.}

El esguince de tobillo es una de las lesiones ortopédicas más comunes para la vida diaria de personas sean deportistas o no. Normalmente el diagnóstico es subestimado por el mismo lesionado sin considerar que las lesiones en los ligamentos producen inestabilidad en la postura siempre que no se haga el correcto tratamiento de la lesión.

Cuando se hace evidente una afección física, el paciente siempre espera el "mejor escenario" para el tratamiento y consecutiva recuperación. Esta esperanza es confundida con la posibilidad de acceder al "tratamiento conservador", pero ¿de qué se trata el mismo?

La discusión respecto al uso de la terminología médica la hemos conseguido en una editorial escrita por William Bakeley (1971) en donde expone su inquietud y aclara finalmente el uso correcto, según su criterio, de la terminología "tratamiento conservador", a continuación, señala:

"Hace unos pocos años se señaló que los Cirujanos estaban abusando de la palabra "cirugía", nombre que describe un campo de especialización médica y que comprende el diagnóstico de la enfermedad, la preparación preoperatoria, la operación y el cuidado postoperatorio hasta la recuperación. Por consiguiente, se consideró impropio e incorrecto decir que un paciente "es llevado a cirugía" o que va a tener su "cirugía" en un tiempo dado. Correctamente se diría que él va a ser llevado a un servicio quirúrgico o que va a ser intervenido quirúrgicamente en determinada fecha y que su cirugía incluirá cuidados y filosofía mayor que estos eventos aislados...Es tiempo de usar el 


\section{Tratamiento conservador del esguince de tobillo}

Vol. 3, núm. 3 Esp., (2019)

Joao Andrés Rodríguez Molina; Pedro Javier Chong Cevallos; Julio César Tixe Peralta; Rogelio

Andrés Leyton Acuña

mismo mecanismo para erradicar de las comunicaciones médicas la anticuada frase de "tratamiento conservador". La implicación del tratamiento conservador es clara para el médico por el uso prolongado y por su primitivo origen en una época en que una intervención quirúrgica era el último recurso de tratamiento. Pero ¿cuál es la interpretación actual para el profano, para el estudiante de Medicina y para el joven médico?...” (Bakeley, 1971).

En el presente trabajo se pretende encarar esta idea "no invasiva" y sus reales implicaciones en el tratamiento de esguince de tobillo a través de una revisión bibliográfica selectiva de la literatura académica disponible al respecto.

\section{Metodología.}

Se trata de una colección analítica académica de bibliografía que nos permite construir un artículo referencial para conocer el diagnóstico y tratamiento necesario para la recuperación correcta de las lesiones de esguince de tobillo. Utilizando el motor de búsqueda google académico y Pub Med para conseguir dichas publicaciones.

\section{Resultados.}

Es oportuno cerrar la incógnita planteada en la introducción respecto al correcto uso del término "tratamiento conservador" visto que, para el caso que nos atañe, es importante aclararlo. Para ello, continuamos con la exposición de Bakeley (1971) por considerarla correcta y precisa, continúa entonces exponiendo que: 


\section{Tratamiento conservador del esguince de tobillo}

Vol. 3, núm. 3 Esp., (2019)

Joao Andrés Rodríguez Molina; Pedro Javier Chong Cevallos; Julio César Tixe Peralta; Rogelio Andrés Leyton Acuña

“...En el tiempo presente el uso de la palabra "conservador" para indicar un tratamiento no operatorio o médico no sirve para ninguno de estos propósitos. La lectura cuidadosa de diccionarios, médicos y no médicos, revela unanimidad de definición para la palabra conservador: "moderado, seguro, para preservar con cuidado, para conservar intacto o no modificado" y en un sentido médico "que lleva por mira la preservación de la salud o la restauración y reparación de partes o funciones, opuesto a medidas radicales o heroicas de tratamiento y fijo a principios sólidos, no especulativos". ¿Cree el competente y consciente cirujano que recomienda un tratamiento quirúrgico, que su recomendación es inmoderada, especulativa o es irrazonablemente peligrosa? ¿Es el término "conservador" remotamente sinónimo con "no operatorio'" en el presente estado del arte? ... Estos absurdos son usados para enfatizar que tratamiento conservador no es necesariamente sinónimo con tratamiento no operatorio y que, en muchos ejemplos, es más bien sinónimo de tratamiento operatorio. Se sugiere que los términos "operatorio" y "no operatorio", "médico" y "quirúrgico" son conceptualmente correctos y representan más precisamente nuestro empeño, que es aplicar los medios apropiados de tratamiento en el momento indicado. Por consiguiente, el tratamiento es invariablemente conservador, se efectúe o no una intervención quirúrgica, y a menudo precisamente por ello. (Bakeley, 1971).

La lesión de los ligamentos del tobillo es el traumatismo que ocurre más frecuentemente durante las actividades de la vida diaria y de la práctica deportiva. A pesar de la frecuencia de estas lesiones, las técnicas y los métodos de tratamiento varían ampliamente, probablemente 


\section{Tratamiento conservador del esguince de tobillo}

Vol. 3, núm. 3 Esp., (2019)

Joao Andrés Rodríguez Molina; Pedro Javier Chong Cevallos; Julio César Tixe Peralta; Rogelio

Andrés Leyton Acuña

porque su biomecánica y la valoración de la integridad anatómica no están completamente entendidas. Con seguridad también a ello se deba la frecuente inestabilidad crónica como secuela El esguince de tobillo es la lesión de los ligamentos alrededor del mismo; se clasifica dependiendo del grado de severidad y las estructuras afectadas. El mecanismo de lesión se relaciona con posiciones en inversión o eversión, por lo que es común que ocurra durante prácticas deportivas. Sus principales manifestaciones son el dolor, el edema y la limitación en diversos grados para la marcha (Pérez, y otros, 2004).

Figura 1. Vista Lateral y media del tobillo

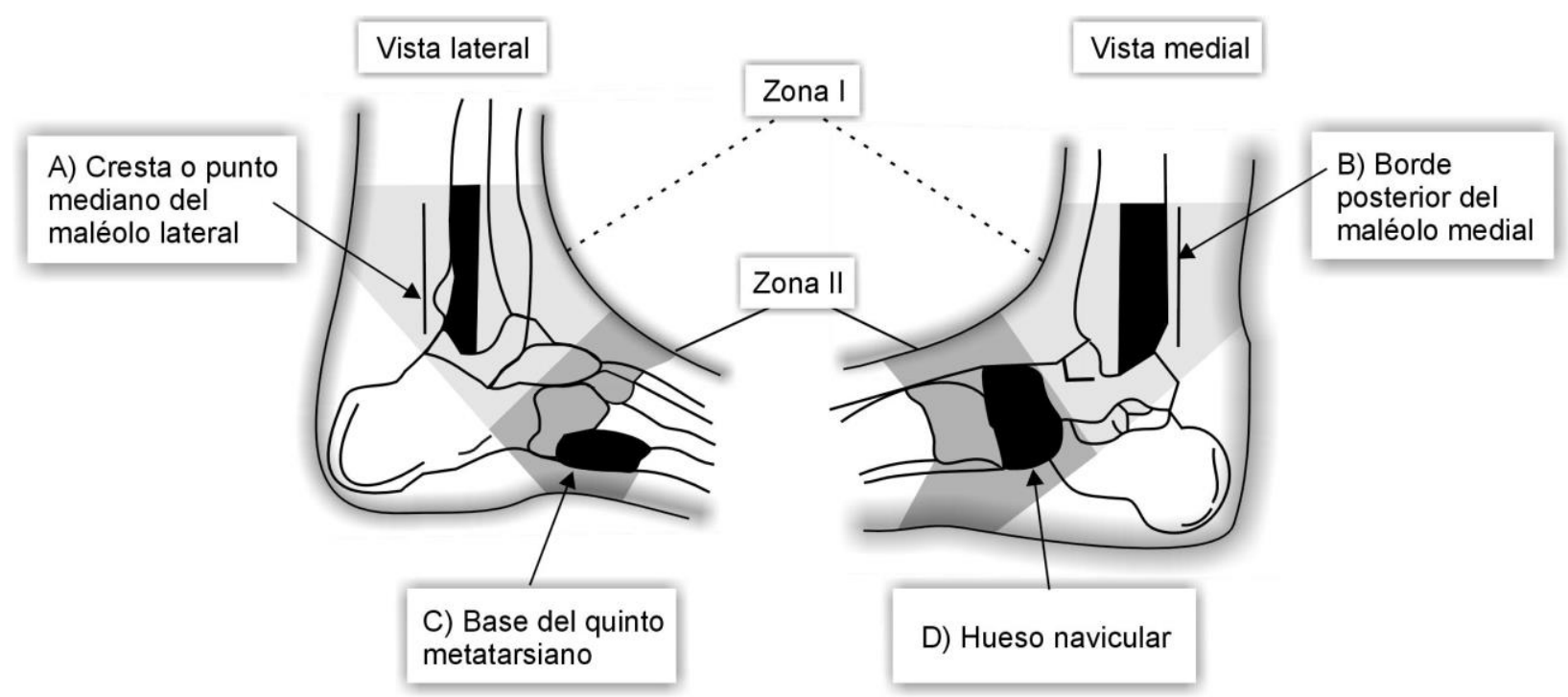

Fuente: (Pérez, y otros, 2004)

Casi todos los médicos y muchos profanos consideran el esguince de tobillo como una lesión trivial, sin importancia a pesar de que el $75 \%$ de los casos existe ruptura de uno o varios ligamentos. O’Donoghue (1970) define que el esguince se puede clasificar en Grado I (Leve): 


\section{Tratamiento conservador del esguince de tobillo}

Vol. 3, núm. 3 Esp., (2019)

Joao Andrés Rodríguez Molina; Pedro Javier Chong Cevallos; Julio César Tixe Peralta; Rogelio Andrés Leyton Acuña

desgarro de algunas fibras con escasa hemorragia y no hay evidencia clínica de perdida funcional; Grado II (Moderado): existe alguna pérdida funcional debido a la ruptura parcial del ligamento y Grado II (Severo): Existe daño completo y pérdida total de la función que pudiera comprometer 1, 2 o 3 ligamentos del complejo lateral o medial del tobillo. (Gerstner \& Rojas, 1979).

Los esguinces de la articulación subastragalina, lesiones osteocondrales del astrágalo, lesiones del nervio peroneal y lesiones de ramas del nervio peroneo superficial, pueden ir asociados a lesiones del ligamento lateral externo, pudiendo pasar desapercibidos en el momento de ser visitados en urgencias, lo cual puede provocar secuelas como la inestabilidad crónica del tobillo, llegando a cifras del $20 \%$. La actitud conservadora ha demostrado mejores resultados que la intervención quirúrgica, y es el tratamiento funcional el que mayores resultados ha evidenciado (Guirau, Pleguezuelos, \& Mesquida, 2004).

Son muchas las lesiones de la articulación del tobillo que se parecen al esguince de tobillo, incluyendo neoplasias como el osteoma osteoide, granuloma eosinófilico y la sinovitis pigmentada vellonodular (Figura 2). Snyder et al.3 y O`Neil y Micheli4 informaron de que existía una alta incidencia de coaliciónes tarsales (Figura 3) asociadas con esguinces de tobillo en niños y adolescentes, incluyendo las coaliciones fibrosas, coaliciones cartilaginosas y coaliciones óseas. La fractura osteocondral también puede parecerse al esguince de tobillo. Cuando existen síntomas de esguince de tobillo que no se resuelven al cabo de las 4 o 5 semanas de tratamiento conservador, es recomendable valorar una tomografía computarizada y una evaluación adicional para descartar coalición tarsalo fractura osteocondral del astrágalo. (Muñoz \& Navarro, 2015). 


\section{Tratamiento conservador del esguince de tobillo}

Vol. 3, núm. 3 Esp., (2019)

Joao Andrés Rodríguez Molina; Pedro Javier Chong Cevallos; Julio César Tixe Peralta; Rogelio Andrés Leyton Acuña

Figura 2. La sinovitis pigmentada vellonodular erosiona la porción superior del cuello del astrágalo causado síntomas de esguince de tobillo en atletas jóvenes.

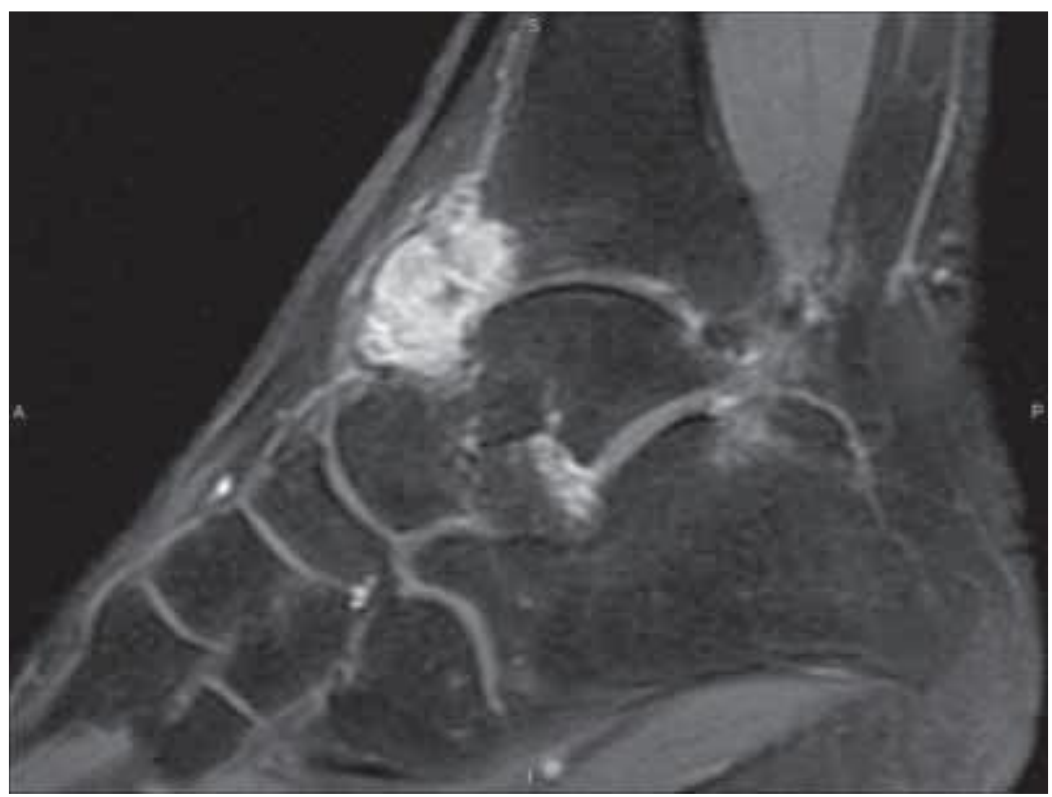

Fuente: (Muñoz \& Navarro, 2015)

Figura 3. La tomografía computarizada muestra una coalición tarsal entre astrágalo y calcáneo
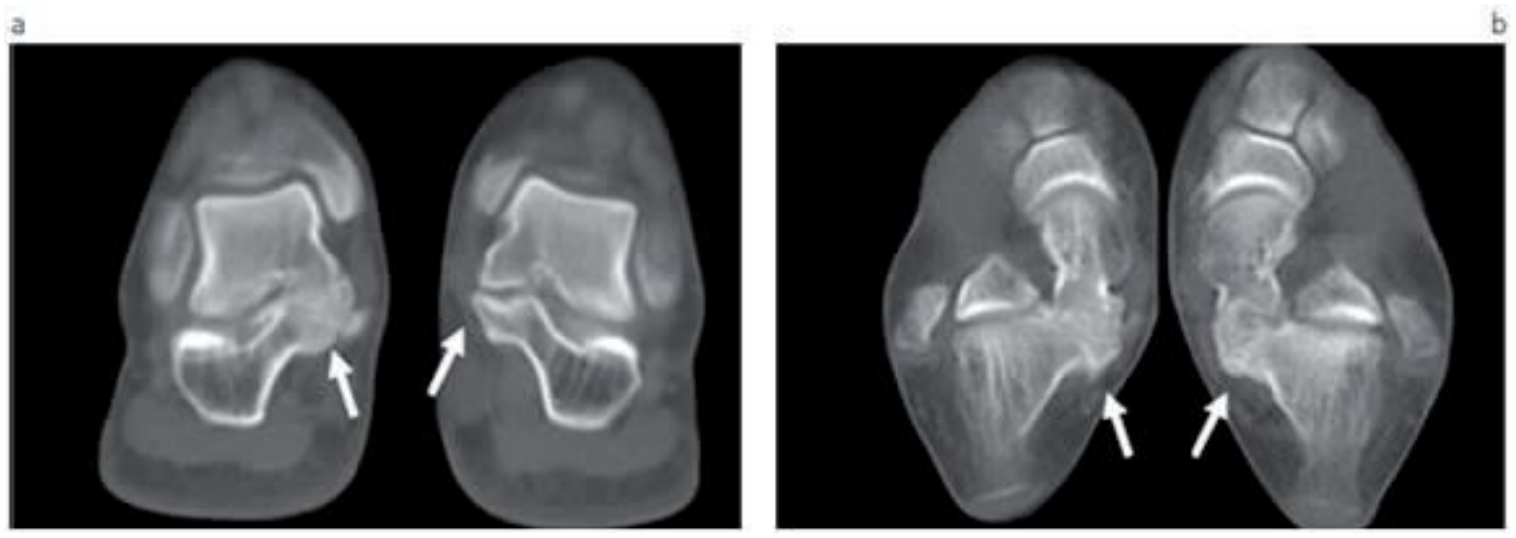

Fuente: (Muñoz \& Navarro, 2015) 


\section{Tratamiento conservador del esguince de tobillo}

Vol. 3, núm. 3 Esp., (2019)

Joao Andrés Rodríguez Molina; Pedro Javier Chong Cevallos; Julio César Tixe Peralta; Rogelio Andrés Leyton Acuña

La restricción de actividad física es por lo general suficiente como tratamiento en los pacientes más jóvenes, aunque los pacientes que están en etapas más cercanas e incluso en la adolescencia pueden combinar también un medicamento anti-inflamatorio o una inyección de cortisona. Rara vez es necesaria la artroscopia o artrotomía para la escisión de un espolón óseo o de osteofitos distales de la tibia o cuello del astrágalo (Figura 4). (Muñoz \& Navarro, 2015).

Figura 4. La radiografía lateral muestra una exóstosis distal y lateral tibial.

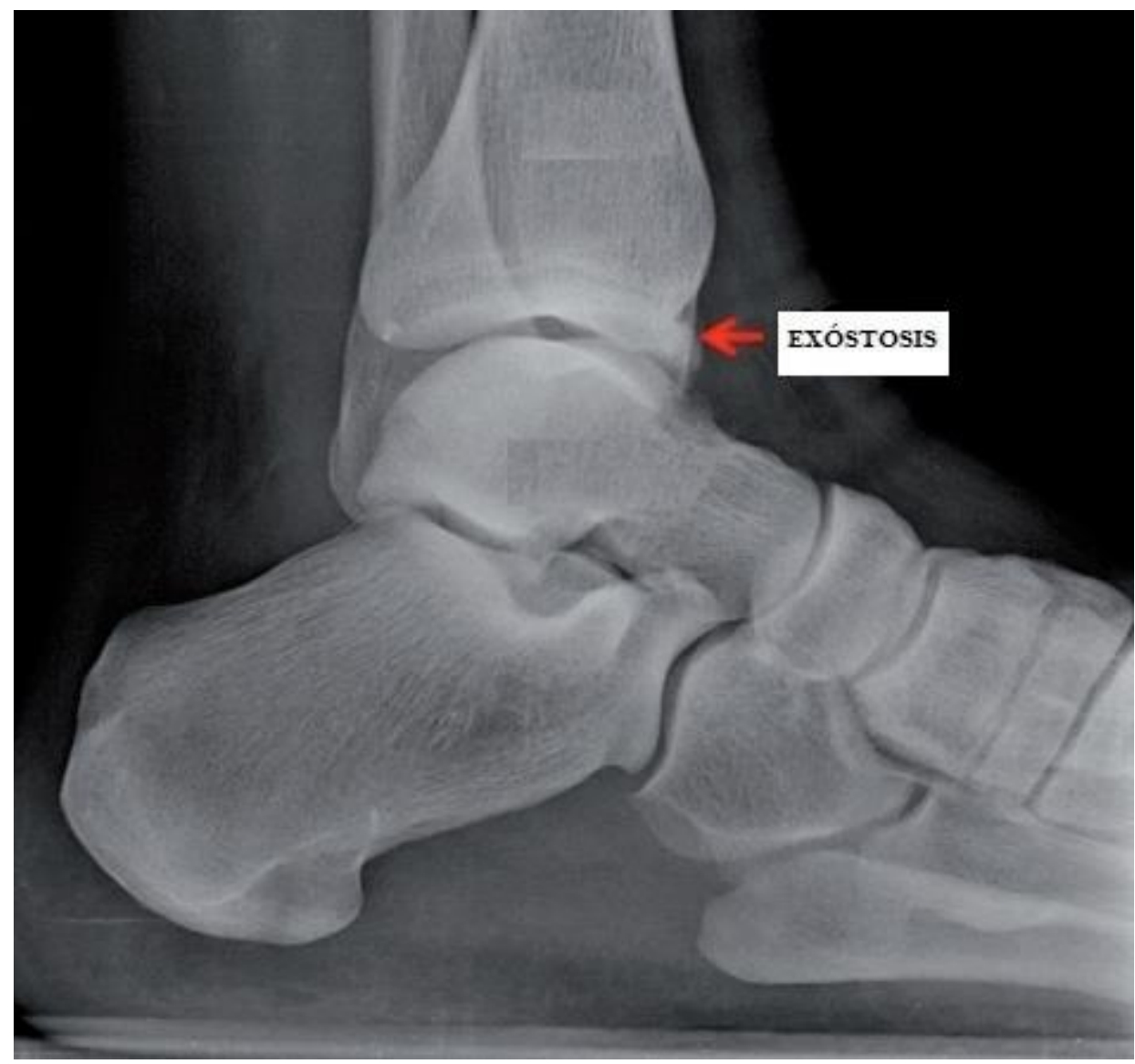

Fuente: (Muñoz \& Navarro, 2015)

Se ha encontrado otras maneras de clasificar los esguinces 


\section{Tratamiento conservador del esguince de tobillo}

Vol. 3, núm. 3 Esp., (2019)

Joao Andrés Rodríguez Molina; Pedro Javier Chong Cevallos; Julio César Tixe Peralta; Rogelio

Andrés Leyton Acuña

La propuesta por Cass y Morrey:

- Grado I: existe una distensión ligamentosa sin rotura, no hay laxitud y el dolor o los signos inflamatorios son ligeros.

- Grado II: existe una rotura parcial, podemos encontrar algún grado de laxitud, el dolor y los signos inflamatorios son moderados.

- Grado III: rotura completa del ligamento e inestabilidad de la articulación; los signos inflamatorios y el dolor son importantes. También encontramos hemorragia y pérdida de la funcionalidad y movilidad del tobillo. (Guirau, Pleguezuelos, \& Mesquida, 2004).

Tabla 5. Clasificación del grado de lesión de los esguinces de tobillo de West Point

\begin{tabular}{llll}
\hline Criterio & \multicolumn{1}{c}{ Gradol } & \multicolumn{1}{c}{ Grado II } & \multicolumn{1}{c}{ Grado III } \\
\hline Localización del dolor & PAA & PAA, CP & PAA, CP, PAP \\
Edema, equimosis & Poco, local & Moderado, local & Significativo, difuso \\
Capacidad para cargar peso & Completa o parcial & Dificultad sin muletas & Imposible sin dolor importante \\
Afectación ligamentosa & Estiramiento & Rotura parcial & Rotura completa \\
Inestabilidad & No & No o poco importante & Si \\
\hline
\end{tabular}

PAA: ligamento peroneo astragalino anterior; CP: ligamento calcaneoperoneo; PAP: ligamento peroneo astragalino posterior.

Fuente: (Guirau, Pleguezuelos, \& Mesquida, 2004)

Para su diagnóstico Pérez y otros (2004) sugieren que sólo se requieren estudios radiológicos si hay dolor en la zona maleolar y uno o más de los siguientes datos:

Una serie radiográfica de tobillo (anteroposterior, lateral y vista de la mortaja) debe ser obtenida al encontrar dolor en la zona maleolar (zona I) y cualquier de los siguientes agregados: 


\section{Tratamiento conservador del esguince de tobillo}

Vol. 3, núm. 3 Esp., (2019)

Joao Andrés Rodríguez Molina; Pedro Javier Chong Cevallos; Julio César Tixe Peralta; Rogelio Andrés Leyton Acuña

- Dolor a la palpación en la cresta o zona media del maléolo lateral

- Dolor a la palpación a lo largo del borde posterior o punto medio del maléolo medial

- Incapacidad para descargar peso sobre el pie afectado durante la exploración

Una serie radiográfica de pie únicamente se requiere si existe dolor en la zona II y cualquiera de los siguientes agregados

- Dolor a la palpación de la base del quinto metatarsiano

- Dolor a la palpación de hueso navicular

- Incapacidad para descargar el peso sobre el pie afectado durante la exploración

Debe considerarse el juicio del clínico para solicitar radiografías en pacientes embarazadas, pacientes con lesión de tobillo mayor de 10 días, lesiones aisladas de la piel sin lesión de tejido blando o reingreso por persistencia de sintomatología de un esguince de tobillo. (Pérez, y otros, 2004).

La localización más frecuente de la lesión es el ligamento lateral externo y dentro de los tres haces que constituyen el mismo, el más habitualmente afectado es el anteromaleolar o ligamento peroneo-astragalino anterior. A pesar de tratarse de la localización más frecuente es la que presenta un mejor pronóstico. La afectación de los ligamentos peroneo-calcáneo y peroneoastragalino posterior son menos habituales, pero presentan, especialmente este último, peor pronóstico. La mayor afectación del ligamento lateral externo está condicionada por la tendencia supinadora en el apoyo inicial del retropié, que suele ser el momento en el que se produce la lesión. En ocasiones, el mecanismo de la propiocepción intenta compensar el movimiento lesivo, 


\section{Tratamiento conservador del esguince de tobillo}

Vol. 3, núm. 3 Esp., (2019)

Joao Andrés Rodríguez Molina; Pedro Javier Chong Cevallos; Julio César Tixe Peralta; Rogelio

Andrés Leyton Acuña

lo que conduce a la distensión ligamentosa mediante una contracción pronadora. Cuando se produce este hecho de manera violenta puede acaecer un esguince combinado del ligamento lateral interno. La lesión aislada del ligamento lateral interno es rara y solamente suele aparecer durante la práctica deportiva de intensidad. (Egocheada, Gonzalez, Montoliu, Rodríguez, \& Del Valle, 2005).

En general, existe consenso en cuanto a que el tratamiento funcional, con sus distintas modalidades, es el más apropiado para el tratamiento de los esguinces grado I y II. En los esguinces grado III, se aconseja reservar el tratamiento quirúrgico para pacientes deportistas o con alta demanda funcional5 y tratar al resto ortopédicamente. Estudios recientes y similares al nuestro, realizados en nuestro país, concluyen que el tratamiento funcional en esguinces grado III comparado con la inmovilización es seguro, se asocia a una más rápida recuperación y está indicado incluso en deportistas. Asimismo, una reciente revisión sistemática del grupo Cochrane concluye que el tratamiento funcional con movilización y carga precoz parece obtener mejores resultados que la inmovilización clásica (Parrón, y otros, 2006).

En primera instancia, el tratamiento de la inestabilidad crónica debe ser conservador, mediante un programa de rehabilitación funcional que incluya fisioterapia, entrenamiento neuromuscular enfocado en la rehabilitación propioceptiva y fortalecimiento muscular. En los casos en los que falle, se indica la reparación quirúrgica. (Reyes, Eckholt, González, \& Poggio, 2019).

El objetivo de la cirugía es restablecer la estabilidad articular y reducir el riesgo de futuros esguinces de tobillo, disminuyendo de esta manera el daño al cartílago. Se han descrito 


\section{Tratamiento conservador del esguince de tobillo}

Vol. 3, núm. 3 Esp., (2019)

Joao Andrés Rodríguez Molina; Pedro Javier Chong Cevallos; Julio César Tixe Peralta; Rogelio Andrés Leyton Acuña

más de 70 procedimientos quirúrgicos para el tratamiento de la inestabilidad crónica de tobillo, los cuales se dividen en técnicas de tenodesis o no anatómicas (Evans, Watson-Jones, ChrismanSnook) y técnicas anatómicas con y sin aumentación (Broström, Karlsson, Broström-Gould). En la literatura frecuentemente se menciona el uso del retináculo extensor inferior o periostio como refuerzo de tejido nativo para la reparación anatómica. En los casos en los que se indica reparación anatómica con refuerzo de tejido nativo y en los que durante la cirugía estas estructuras sean insuficientes o no se logren identificar, los autores pretenden mostrar una alternativa de tejido nativo como técnica de aumentación, utilizando el fascículo distal del ligamento tibioperoneo anteroinferior (LTPAI) para la reparación de la inestabilidad lateral crónica de tobillo. (Reyes, Eckholt, González, \& Poggio, 2019).

\section{Conclusiones.}

Hablar de un tratamiento conservador, tal como ha sido expuesto al inicio de la investigación y como lo aclara Bakeley (1971) no exime al médico de hoy a utilizar el término como algún modismo del lenguaje. Sin embargo, hemos reafirmado que un tratamiento conservado no necesariamente exime de una intervención quirúrgica.

El 85\% de los esguinces son consecuencia de un movimiento forzado de inversión. Se sabe que más del $80 \%$ recidivan y que hasta un $40 \%$ pueden acabar en inestabilidad crónica que conlleva debilidad muscular, laxitud ligamentosa y déficits propioceptivos y de control postural condicionando el desarrollo de la actividad deportiva e incluso de la actividad cotidiana. El estudio del equilibrio postural después del esguince ayuda a conocer una parte de las secuelas de esta lesión y nos ofrece información para mejorar la recuperación, disminuir las recidivas e 


\section{Tratamiento conservador del esguince de tobillo}

Vol. 3, núm. 3 Esp., (2019)

Joao Andrés Rodríguez Molina; Pedro Javier Chong Cevallos; Julio César Tixe Peralta; Rogelio

Andrés Leyton Acuña

incluso poder prevenir los esguinces en personas que no los hayan padecido nunca. (Martín \& Aguado, 2011).

Por ser una lesión tan común cuando, a través de tratamientos conservadores de inmovilización, vendajes, masajes y ungüentos es notorio el alivio pero a largo plazo la lesión sigue frágil y es cuando deviene una proclama común "esguince primero, esguince para toda la vida", se trata entonces de evitar este sentir popular enfrentando al paciente a una realidad y es que, no hay mejoría absoluta si a través del tiempo el paciente es propenso a otras afecciones en el tobillo, pues deriva finalmente en lesiones graves.

El empleo de bota de escayola como tratamiento de esguinces lleva aparejado toda una serie de efectos secundarios de importante señalización como son la pérdida de propiocepción, las atrofias musculares, la rigidez articular, así como cierto grado de impotencia funcional, especialmente problemática y a señalar en pacientes deportistas. La pérdida de propiocepción asociada a la atrofia muscular por desuso condiciona y facilita la aparición de hiperlaxitud ligamentosa, causa frecuente del esguince crónico que no esguinces de repetición, ya que el problema radica y se encuentra en un primer y único esguince que, a nuestro modo de ver, ha sido mal tratado. Por lo anterior, abogamos por evitar la práctica habitual de colocación de botas de yeso en estos pacientes, reservando su uso exclusivamente para esguinces graves. (Egocheada, Gonzalez, Montoliu, Rodríguez, \& Del Valle, 2005).

Cuando un esguince de tobillo no se resuelve, debe tenerse en cuenta la posibilidad de que exista una fractura osteocondral de astrágalo. Baker et al (1986) informaron que el $80 \%$ de los pacientes con osteocondritis disecante traumáticas tienen antecedentes de esguince de tobillo 


\section{Tratamiento conservador del esguince de tobillo}

Vol. 3, núm. 3 Esp., (2019)

Joao Andrés Rodríguez Molina; Pedro Javier Chong Cevallos; Julio César Tixe Peralta; Rogelio Andrés Leyton Acuña

aparentemente benigno. Si hay derrame persistente, sinovitis retardada o bloqueo de la articulación, el examen radiográfico debe hacerse 4 o 5 semanas después de la lesión. La proyección oblicua y en flexión que eviten la superposición tibial y generalmente muestran la lesión con mayor claridad que las radiografías simples. Si se sospecha fractura osteocondral debe hacerse una gammagrafía ósea. Una vez que la lesión está localizada, las tomografías pueden determinar su profundidad y tamaño. La resonancia magnética es, en la actualidad, cara y probablemente no sea necesaria; sin embargo, se puede determinar si el fragmento está unido o no al cráter. (Muñoz \& Navarro, 2015).

Finalmente, el tratamiento siempre será conservador cuando el diagnóstico es el adecuado. Independientemente de que las lesiones más graves requieran de una intervención quirúrgica está visto que alternativas como las fisioterapias, la inmovilización temprana siempre serán parte del plan médico para la recuperación del paciente preventivamente para evitar la cirugía o precisamente para recuperarse de ella.

\section{Bibliografía.}

Bakeley, W. (1971). ¿Qué es Tratamiento Conservador? Surgery, Gyn. \& Obst, 132(687), 272273.

Egocheada, J., Gonzalez, V., Montoliu, M., Rodríguez, B., \& Del Valle, M. (2005). Propuesta de protocolo para tratamiento de esguinces de tobillo. Semergen, 31(4), 161-163.

Gerstner, J., \& Rojas, G. (1979). Esguinses de tobillo. Diagnóstico y tratamiento. Acta Medica Valle, 10(1), 12-17.

Guirau, L., Pleguezuelos, E., \& Mesquida, M. (2004). Tratamiento funcional del esguinse de tobillo. Rehabilitación (Madr), 38(4), 182-187. 


\section{Tratamiento conservador del esguince de tobillo}

Vol. 3, núm. 3 Esp., (2019)

Joao Andrés Rodríguez Molina; Pedro Javier Chong Cevallos; Julio César Tixe Peralta; Rogelio

Andrés Leyton Acuña

Martín, L., \& Aguado, X. (2011). Revisión de las repercusiones de los esguinces de tobillo sobre el equilibrio postural. Apunts Medicinas de L'Esport, 46(170), 97-105.

Muñoz, J., \& Navarro, J. (2015). Lesiones de astrágalo que pueden ser confundidas con esguinces de tobillo. Revista Internacional de Ciencias Podológicas, 9(2), 106-116.

Parrón, R., Barriga, A., Herrera, J., Pajares, S., Gómez, R., \& Poveda, E. (2006). Inmovilización frente al tratamiento funcional de esguinces de tobillo grado III. Archivos de Medicina del Deporte, 23(111), 10-16.

Pérez, J., Hernandez, E., Mazadiego, M., Mora, R., Rangel, Y., Sánchez, R., . . . López, V. (2004). Guía clínica para la atención del paciente con esguinse de tobillo. Revista Médica del IMSS, 5(42), 437-444.

Reyes, C., Eckholt, S., González, J., \& Poggio, D. (2019). Método de aumentación en reparacion de inestabilidad lateral crónica de tobillo: consejo técnico. Revista del pie y tobillo, 33(1), 2173-2949.

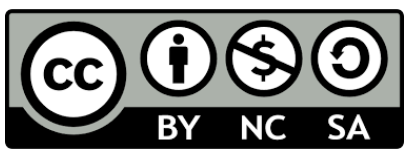

RECONOCIMIENTO-NOCOMERCIAL-COMPARTIRIGUAL

CC BY-NC-SA

ESTA LICENCIA PERMITE A OTROS ENTREMEZCLAR, AJUSTAR Y CONSTRUIR A PARTIR DE SU OBRA CON FINES NO

COMERCIALES, SIEMPRE Y CUANDO LE RECONOZCAN LA AUTORÍA Y SUS NUEVAS CREACIONES ESTÉN BAJO UNA LICENCIA CON LOS MISMOS TÉRMINOS. 